\title{
Quiste aracnoideo espinal epidural postraumático: presentación de un caso
}

\author{
O. Hernández-León; F.R. Pérez-Nogueira y N. Corrales
}

Servicio de Neurocirugía. Hospital Universitario “Abel Santamaría Cuadrado”. Pinar del Río. Cuba.

Resumen

Introducción. Los quistes aracnoideos extradurales espinales son lesiones poco frecuentes. Clínicamente se caracterizan por un cuadro mielopático progresivo, asociado o no a crisis radiculares. Para su diagnóstico radiológico las técnicas de resonancia magnética actuales posibilitan definirlos adecuadamente y conocer su localización topográfica. Los antecedentes patológicos del paciente son esenciales para establecer se etiología encontrándose en muchos casos una historia de trauma espinal, cirugía y menos frecuentemente anomalías congénitas asociadas. El tratamiento en la mayoría de los casos es quirúrgico.

Caso clínico. Presentamos el caso de un paciente de 35 años con antecedentes de una hemisección medular dorsal por arma blanca hace dos años. Evolutivamente se deterioran sus funciones motoras presentándose con una paraparesia espástica. Los estudios radiológicos mostraron la presencia de la lesión quística extradural comprimiendo el cordón medular a nivel dorsal (D3D4). Se realizó cirugía descompresiva y se identificó un desgarro meníngeo a ese nivel que fue suturado. La evolución postoperatoria fue satisfactoria.

Conclusiones. El tratamiento quirúrgico efectivo de estas lesiones está dado por la detección del defecto meníngeo y su cierre.

PALABRAS CLAVE. Trauma raquimedular. Quiste aracnoideo espinal. Médula espinal dorsal. Compresión medular

Postraumatic epidural arachnoid spinal cyst: case report

Summary

Introduction. Extradural arachnoid spinal cysts are unfrequent lesions that are associated with spinal

Recibido: 23-06-40. Aceptado: 4-12-10 trauma, surgery and less frequently with congenital anomalies. The clinical manifestations are similar to those seen with other compressive spinal cord lesions. Magnetic resonance techniques allow to diagnose correctly this pathology and to define its thopographic situation. The pathologic history of the patient is essencial to establish the ethiology. Surgery is the elective treatment in most cases.

Clinical case. The patient is a 35 years old man who has a medical history of penetrating spinal trauma two years ago. In that instance he suffered an unilateral spinal cord section at D2-D3 level with the corresponding Brown Sequard syndrome. A small wound was detected at the skin dorsal level and it was closed without difficulties. At the beginning, he improved his motor right leg function with rehabilitation and vitamins. After two years of good recovery he came to our hospital suffering a neurological deterioration of six months of evolution. The physical examination revealed an spastic paraparesis. Magnetic resonance was performed demonstrating a cystic extradural collection compressing the spinal cord at D3-D4 level. Surgical decompressive treatment allowed to excise the cyst and it was possible to define a dural tear that was closed successfully. The outcome was good with restoration of the initial motor function that he had after the spinal trauma.

Conclusions. Surgical management of postraumatic epidural arachnoid spinal cyst allows to detect the meningeal tear and to close it, which is highly effective on these kinds of lesions.

KEY WORDS. Spinal cord injury. Arachnoid spinal cyst. Dorsal spinal cord. Spinal cord compression.

Introducción

Los quistes aracnoideos extradurales son lesiones raras

Abreviaturas. LCR: líquido cefalorraquideo. RMN: resonancia magnética nuclear. 


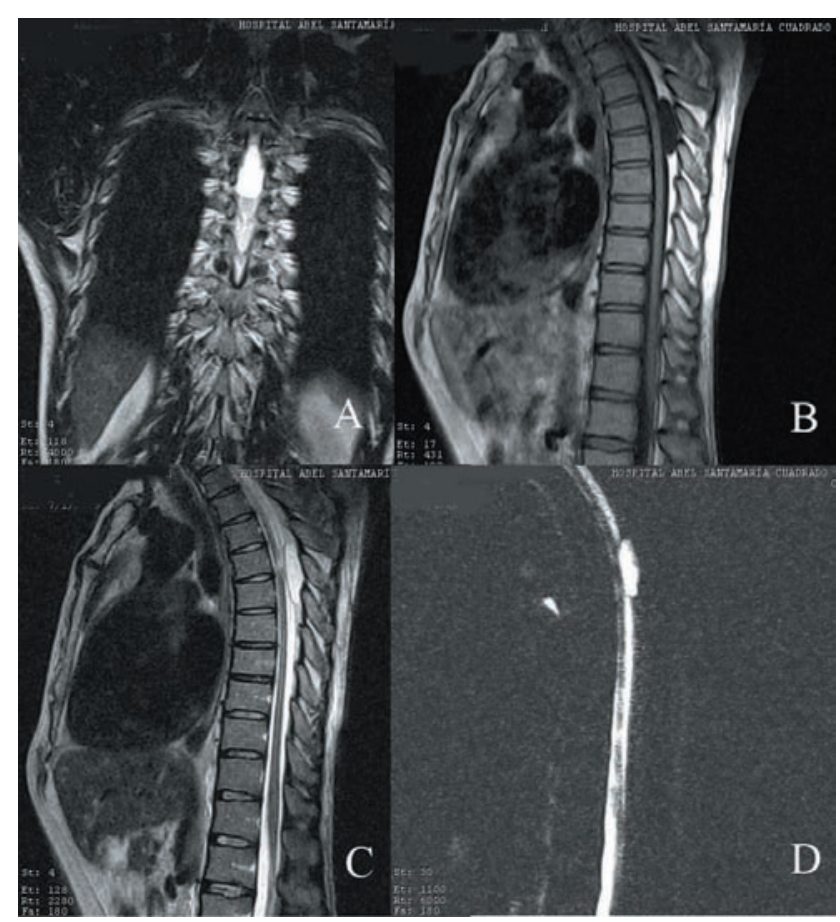

Figura 1. A, B y C. RMN preoperatoria donde se observa una lesión hiperintensa en secuencias de T2 e hipointensa en secuencias de T1, ocupando el canal raquídeo en sentido longitudinal y posterior al saco dural, de bordes bien definidos. D. En la técnica de mielografía se comprueba que la lesión altera la integridad de la columna de LCR.

y poco frecuentes, que se caracterizan por mostrar crecimiento expansivo dentro del canal raquídeo. El neurocirujano debe tener siempre presente esta entidad, pues clínicamente se comporta como un síndrome de compresión medular, que en la mayoría de los casos la cirugía puede mejorar el estado neurológico del paciente al lograr la descompresión raquídea. Esta patología se asocia con traumas, cirugía, fenómenos de aracnoiditis y defectos del
2011; 22: 267-270

tubo neural ${ }^{6}$. En los casos de etiología traumática existe una lesión de la duramadre ${ }^{13}$. Los mecanismos reportados en su etiología incluyen el trauma penetrante, fracturas espinales con desgarros durales por fragmentos óseos y lesiones durales intraoperatorias ${ }^{13,15,17}$. La herniación aracnoidea a través del defecto meníngeo se transforma en una bolsa de tejido conectivo y el crecimiento del quiste progresa debido a las pulsaciones y presión del $\mathrm{LCR}^{14}$.

A continuación, presentamos el caso de un paciente con un quiste aracnoideo dorsal de localización epidural y de etiología traumática tratado en nuestro Servicio, incluyendo una discusión del mismo a partir de una revisión de la literatura científica.

\section{Caso clínico}

Paciente varón, de 35 años, saludable, que hace tres años sufrió un trauma espinal penetrante con un objeto punzante que le ocasionó parálisis motora súbita del miembro inferior derecho Al examinarlo se comprobó una herida de $2 \mathrm{~cm}$, localizada a la derecha de los procesos espinosos de D3 y D4, que no evidenciaba salida de LCR y fue suturada. El examen neurológico detallado permitió diagnosticar un síndrome de Brown Sequard secundario a una hemisección medular derecha. El paciente evolucionó favorablemente con tratamiento rehabilitador, mostrando mejoría notable de la actividad motora lo cual posibilitó la reincorporación a la marcha con apoyo.

A los dos años de ocurrida la lesión el paciente comienza a notar deterioro de la fuerza muscular de ambos miembros inferiores, e impotencia sexual hasta el momento no referida. Durante el examen neurológico se comprueba una paraparesia espástica predominantemente derecha.

En RMN dorsal (figura 1) se identificó la presencia de una lesión quística que topográficamente se corresponde con la lesión espinal antigua, que comprime la médula a ese nivel.

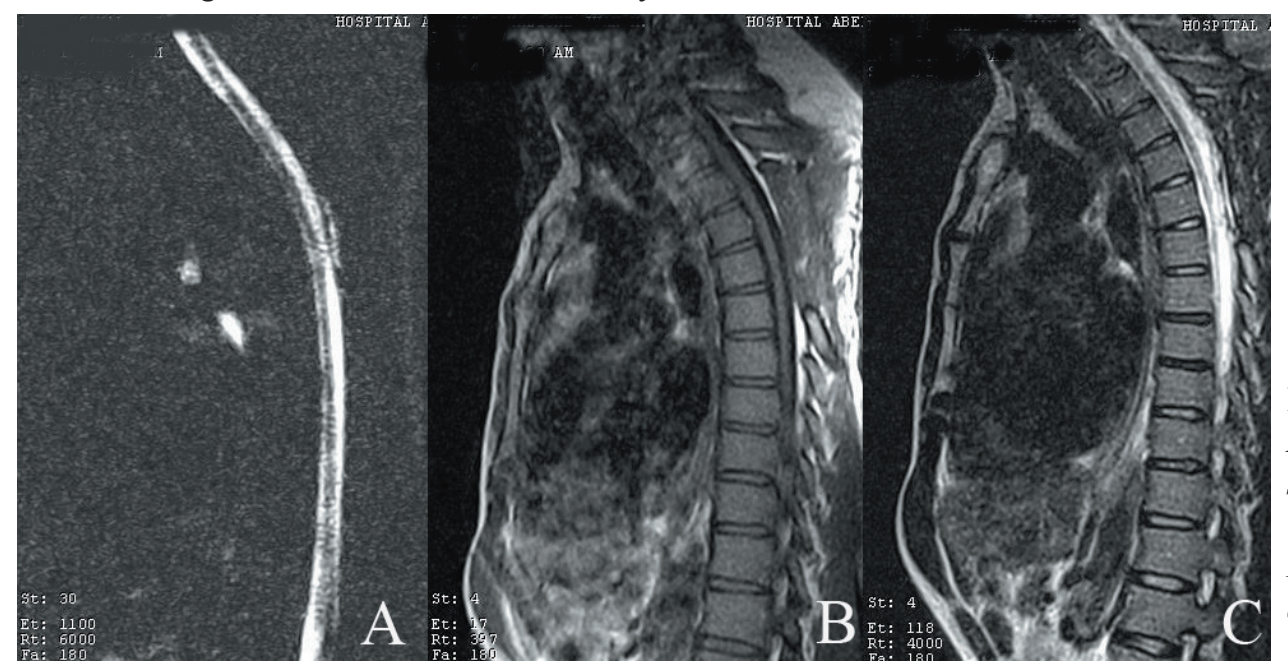

Figura 2. RMN postoperatoria. A. En la técnica de mielografia se observa que se restablece la integridad de la columna de LCR. B y C. En las secuencias de T1 y T2 no se observa la imagen quística. 
Se realizó laminectomía dorsal exponiendo la masa quística cubierta por un tejido transparente de apariencia aracnoidal cuyo contenido era LCR. Se retiró todo este tejido epidural y se expuso un desgarro de duramadre longitudinal de aproximadamente $1 \mathrm{~cm}$. Se realizó disección de los bordes fibróticos del defecto dural, separándolo del área de gliosis medular subyacente con ayuda de magnificación óptica y se suturaron sus bordes.

La evolución del paciente fue satisfactoria, reanudando la marcha en el postoperatorio inmediato con las características que tenía anteriormente. En el estudio radiológico posquirúrgico se comprueba la desaparición de la imagen quística (Figura 2).

\section{Discusión}

Los quistes aracnoideos epidurales son relativamente raros y la patogénesis todavía es confusa. La fisiopatogenia mas frecuentemente invocada es la presencia de un defecto en la duramadre que ocasiona la salida del líquido subaracnoideo y la herniación de la aracnoides, aunque en la literatura revisada se han reportado pacientes donde en la exploración quirúrgica no se encuentra el defecto meníngeo $^{8,9}$. El defecto dural se encuentra más frecuentemente en los casos donde la etiología es traumática, como el nuestro, o postquirúrgica, y juega un papel muy importante, pues si quirúrgicamente se logra su cierre, se soluciona la enfermedad. En los pacientes donde se invoca el origen congénito también puede encontrarse el defecto dura ${ }^{16}$. Esta entidad puede estar presente en el contexto de síndromes de transmisión genética como el síndrome de Klippel-Trénaunay ${ }^{4}$, donde existe un disturbio de origen mesodérmico que pudiera ser el causante del defecto dural. En estos casos de causa genética y/o hereditaria es frecuente las formas de presentación múltiples dentro del canal raquídeo ${ }^{7,18}$, por lo que supuestamente estos pacientes tendrán una alteración importante y difusa de la circulación del líquido cefalorraquídeo cuyo origen embrionario no está bien establecido. Se han publicado dos casos de dos pacientes con un quiste de este tipo en asociación con una espina bífida oculta $^{1}$, lo cual apoya la teoría de que el defecto dural que da origen al quiste se corresponde con una disgenesia dural segmentaria en el contexto de una malformación disrrásfica neuroectodérmica.

Estas colecciones quísticas se localizan más frecuentemente en los segmentos dorsales bajos y dorsolumbar, ocupando la parte dorsal, siendo excepcionales los casos de localización ventral al saco dural ${ }^{12}$. En el caso que presentamos la etiología fue traumática y la localización fue en región torácica alta y dorsal al saco dural en relación con la lesión sufrida años atrás.

El cuadro clínico de estos pacientes en los casos sintomáticos generalmente obedece a un trastorno mielopático, pero pueden estar asociados otros síntomas como el dolor y las crisis radiculares fluctuantes o esporádicas en relación con cambios en las dimensiones quísticas y el patrón de compresión medular por los efectos posturales o cambios de posición corporal que inducen cambios de presiones intrarraquídeas ${ }^{3}$.

El diagnóstico se realizó mediante las diferentes técnicas convencionales de resonancia magnética nuclear para el segmento espinal, como lo muestra la figura. La utilidad de este estudio radiológico queda bien establecido por las posibilidades que ofrece en la adecuada definición anatómica de estas lesiones. En instituciones que cuentan con tecnología de RMN más avanzada en ocasiones es posible identificar el sitio del defecto dural mediante técnicas dinámicas ${ }^{10,11}$, con las cuales ha sido posible observar las modificaciones que sufren estos quistes en las dimensiones y el patrón de compresión medular durante maniobras posturales que se le ordenan realizar al paciente ${ }^{3}$.

Las indicaciones quirúrgicas de estas lesiones están dadas sobre todo para obtener alivio y regresión del cuadro de compresión medular, pues al eliminar las membranas que rodean esta cavidad quística se proporciona la descompresión del canal raquídeo. Así mismo en los casos, como el nuestro en los que existe una elevada posibilidad de que exista un defecto dural, la detección de éste y el cierre de la duramadre, evitan la reaparición de la lesión expansiva y por lo tanto soluciona el problema de manera definitiva. Hay autores que ponen en consideración el tratamiento quirúrgico en casos en los cuales sólo se observan crisis intermitentes de dolor de causa espinal sin lesión neurológica y proponen el seguimiento clínico de estos pacientes mientras no aparezcan signos de deterioro neurológico ${ }^{5}$. Nosotros recomendamos el tratamiento quirúrgico de todos los quistes aracnoideos epidurales en los que exista un antecedente causal traumático y presumiblemente exista un defecto dural para evitar complicaciones mayores como el deterioro clínico del paciente e incluso la herniación medular a través del sitio del desgarro dural la cual ha sido reportada en asociación con esta entidad ${ }^{2}$. En estos casos la angulación del cordón medular alteraría la vascularidad de la médula y la arquitectura de los fascículos que contiene, ocasionando efectos que pudieran evolucionar de forma irreversible.

\section{Conclusiones}

Los quistes aracnoideos epidurales postraumáticos son una entidad poco frecuente, que el neurocirujano debe tener en cuenta frente a un paciente con deterioro neurológico, que tenga como antecedente un trauma espinal por arma blanca. El objetivo de la cirugía en esta entidad radica en el cierre efectivo del defecto dural. 


\section{Bibliografia}

1. Apel, K., Sgouros, S.: Extradural spinal arachnoid cysts associated with spina bifida occulta. Acta Neurochir (wien). 2006; 148: 221-226.

2. Cheng, M.h.: Intraspinal extradural arachnoid cyst with spinal cord herniation. J Formos Med Assoc. 1996; 95: 712714.

3. Doita, M., Nishida, K., Miura, J., Takada, T., Kurosaka, M., Fujii, M.: Kinematic magnetic resonance imaging of a thoracic spinal extradural arachnoid cyst: an alternative suggestion for exacerbation of symptoms during straining. Spine. 2003; 28: E229-33.

4. Durmaz, R., Oztürk, Z., Denle, E., Ciftci, E., Atasoy, Ma.: Symptomatic foraminal extradural meningeal cyst. Turk Neurosurg. 2009; 19: 91-95.

5. Ergun, E., Bórcek, A.O., Cemil, B., Doğulu, F., Baykaner, M.K.: Should we operate all extradural spinal arachnoid cysts? Report of a case. Turk Neurosurg. 2008; 18: 52-55.

6. Kahraman, S., Anik, I., Gocmen, S., Sirin, S.: Extradural giant multiloculated arachnoid cyst causing spinal cord compression in a child. J Spinal Cord Med. 2008; 31: 306-308.

7. Kanaan, In., Sakati, N., Otaibi, F.: Type I congenital multiple intraspinal extradural cysts associated with distichiasis and lymphedema syndrome. Surg Neurol.2006; 65: 162-166.

8. Kulkarni, A.G., Goel, A., Thiruppathy, S.P., Desai, K.: Extradural arachnoid cysts: a study of seven cases. Br J Neurosurg. 2004; 18: 484-488.

9. Liu, J.K., Cole, C.D., Sherr, G.T., Kestle, J.R., Walker, M.L.: Noncommunicating spinal extradural arachnoid cyst causing spinal cord compression in a child. J Neurosurg. 2005; 103 (3 Suppl): 266-269.

10. Miyamoto, M., Kim, K., Matsumoto, R., Isobe, M., Isu, T.: Utility of preoperative magnetic resonance imaging myelography for identifying dural defects in patients with spinal extradural arachnoid cysts: case report. Neurosurgery. 2006; 59 (4).

11. Neo, M., Koyama, T., Sakamoto, T., Fujibayashi, S., Nakamura, T.: Detection of a dural defect by cinematic magnetic resonance imaging and its selective closure as a treatment for a spinal extradural arachnoid cyst. Spine. 2004; 29: E426-430.

12. Prevedello, D.M., Tatsui, C.E., Koerbel, A., Grande, C.V., Cordeiro, J.G., Araújo, J.C.: Ventral extradural spinal meningeal cyst causing cord compression: neurosurgical treatment. Arq Neuropsiquiatr. 2005; 63: 855-858.

13. Rawlings, C.E., Nashold, B.S.: Traumatic extradural spinal cyst: a case report and review of the literature. $\mathrm{Br} \mathrm{J}$ Neurosurg. 1989; 3: 403-408.

14. Sinson, G., Sutton, L.N.: Intraspinal Cysts. Cap 185. In: Tindall GT, Cooper PR, Barrow DL. The Practice of Neurosurgery. V-3. New York: William and Wilkins; 1996.p. 2791-2804.

15. Sklar, E., Quencer, R.M., Green, B.A.: Acquired spinal subarachnoid cysts: evaluation with MR, CT myelography and intraoperative sonography. AJNR 1989; 10: 1097-1104.

16. Tureyen, K., Senol, N., Sahin, B., Karahan, N.: Spinal extradural arachnoid cyst. Spine J.2009; 9:10-15.

17. Wilkins, R.H., Odom, G.L.: Spinal extradural cysts. In: Vinken PJ, Bruyn GW, eds. Handbook of clinical neurology. New York: Elsevier, 1976; p. 137-75.

18. Yabuki, S., Kikuchi, S.: Multiple extradural arachnoid cysts: report of two operated cousin cases. Spine. 2007; 32: E585-588.

Hernández-León, O.; Pérez-Nogueira, F.R.; Corrales, N.: Quiste aracnoideo espinal epidural postraumático: presentación de un caso. Neurocirugía 2011; 22; 267-270.

Correspondencia: Dra. Odalys Hernández León, Calle Isabel Rubio \# 210, entre Frank País y Segunda, ciudad de Pinar del Río, Pinar del Río, Cuba, Código Postal 20400. 